\title{
Pressure-induced bonding and compound formation in xenon-hydrogen solids
}

\author{
Maddury Somayazulu1^, Przemyslaw Dera², Alexander F. Goncharov', Stephen A. Gramsch1, \\ Peter Liermann ${ }^{3}$, Wenge Yang ${ }^{3}$, Zhenxian Liu', Ho-kwang Mao, ${ }^{1,3}$ and Russell J. Hemley'
}

\begin{abstract}
Closed electron shell systems, such as hydrogen, nitrogen or group 18 elements, can form weakly bound stoichiometric compounds at high pressures. An understanding of the stability of these van der Waals compounds is lacking, as is information on the nature of their interatomic interactions. We describe the formation of a stable compound in the $\mathrm{Xe}^{-\mathrm{H}_{2}}$ binary system, revealed by a suite of X-ray diffraction and optical spectroscopy measurements. At $4.8 \mathrm{GPa}$, a unique hydrogen-rich structure forms that can be viewed as a tripled solid hydrogen lattice modulated by layers of xenon, consisting of xenon dimers. Varying the applied pressure tunes the Xe-Xe distances in the solid over a broad range from that of an expanded xenon lattice to the distances observed in metallic xenon at megabar pressures. Infrared and Raman spectra indicate a weakening of the intramolecular covalent bond as well as persistence of semiconducting behaviour in the compound to at least $255 \mathrm{GPa}$.
\end{abstract}

$H$ ydrogen occupies a unique position in the periodic table as a result of its quantum nature and simple electronic structure, and the prediction of unusual chemical, electronic and dynamical properties at very high pressures. There is great interest in the behaviour of hydrogen-rich materials over a broad range of thermodynamic conditions ${ }^{1,2}$. In addition to the novel phenomena predicted in their dense metallic phases ${ }^{3,4}$, the introduction of electronic levels in the bandgap by doping with impurity atoms, thereby facilitating changes in the electronic properties at lower pressures, has been predicted ${ }^{5}$. Xenon has the lowest measured metallization pressure among the rare gas solids, and optical measurements have established bandgap closure and concurrent metallization of xenon at $130-150 \mathrm{GPa}$ (refs 6-8). Evidence for xenon doping of hydrogen in matrix isolation experiments at ambient pressure has been reported ${ }^{9,10}$, but the nature of the bonding in these metastable phases is not well understood. There has been growing interest in the chemistry of xenon to form bulk compounds at high pressures ${ }^{11}$ as well as recognition of the biological effects of xenon at high pressures ${ }^{12}$. During the course of investigating the $\mathrm{Xe}-\mathrm{H}_{2}$ system, we have discovered novel compound formation in $\mathrm{Xe}$ and $\mathrm{H}_{2}$. High-pressure van der Waals compounds formed in simple molecular systems were reported in the early 1990s (refs 13,14). In addition to revealing new compounds involving xenon, our data provide the first experimental signatures of pressure-induced bonding states in these materials.

A series of $\mathrm{H}_{2}-\mathrm{Xe}$ gas mixtures was prepared and loaded into diamond anvil cells using a high-pressure gas loading system. Single crystals of the $\mathrm{H}_{2}$-rich mixtures were grown using a combination of varying pressure and temperature. At $4.1 \mathrm{GPa}$, a xenonrich solid was observed to form. Transitions were optically observed at 4.4, 4.9 and 5.4 GPa with accompanying changes in stoichiometry as inferred from relative volume changes of the phases in the cell (see Supplementary Information). Synchrotron X-ray diffraction data were collected at selected pressures. In the diffraction pattern taken at $4.9 \mathrm{GPa}$, a total of 201 reflections (Fig. 1a) could be indexed on a hexagonal unit cell with cell constants $a=8.654(3) \AA$ and $c=12.357(7) \AA$ and the systematic absences yielded the space group $R 3$. The structure of the xenon sublattice could be successfully solved using direct methods, which resulted in an excellent refinement $\left(R_{1}=3.78 \%\right)$ for 120 unique observed reflections (see Supplementary Information). A remarkable feature of the xenon sublattice is the presence of two different sets of $\mathrm{Xe}-\mathrm{Xe}$ distances. The six xenon atoms in the unit cell are arranged into three $\mathrm{Xe}-\mathrm{Xe}$ pairs oriented along the $c$ axis of the unit cell, giving rise to an array of dimers (Fig. 1b). At $4.9 \mathrm{GPa}$ the distance between the xenon atoms within each pair is $3.875(1) \AA$, whereas the closest $\mathrm{Xe}$-Xe inter-dimer distance is 4.915(1) $\AA$. Remarkably, the Xe-Xe distance in the dimer at this pressure is close to that of neutral dimers in the gas phase ( $3.84 \AA$; ref. 15$)$, which is also close to the nearest-neighbour Xe-Xe distance of $3.83 \AA$ in solid fcc xenon at $5 \mathrm{GPa}$ and room temperature ${ }^{16}$. In comparison, the nearest-neighbour distance at $4 \mathrm{~K}$ (at ambient pressure) determined from X-ray diffraction measurements is $4.34 \AA$ in the LT, fcc phase (ref. 17). The structure of the xenon sublattice determined from an analysis of the X-ray diffraction data at 4.9 and 7.1 GPa remained unchanged across the transitions. The transitions therefore arise from changes in the amount of hydrogen assimilated into the material.

Raman and infrared (IR) spectra provide further information about the nature of the high-pressure phases. A total of five vibron modes for $\mathrm{H}_{2}$ were observed in the Raman spectrum (Fig. 2a), indicating that the $\mathrm{H}_{2}$ molecules are intact. The lowfrequency Raman spectrum is found to be indistinguishable from that of pure solid $\mathrm{H}_{2}$ at the same conditions, indicating rotational disorder of the molecules. No signature of $\mathrm{Xe}-\mathrm{H}$ bonding is observed in the vibrational spectra ${ }^{9}$. A total of five IR-active vibron modes are observed (Fig. 2b). Of these, two are simultaneously Raman and IR active. A $3 \times 3 \times 3$ superstructure based on the hcp lattice of solid hydrogen would give rise to similar activity and result in the appearance of additional vibron bands compared to pure $\mathrm{H}_{2}$ as a result of Brillouin zone folding ${ }^{18,19}$. The IR spectrum shows that the compound remains an insulator at $255 \mathrm{GPa}$.

The stoichiometries of the compounds were estimated as follows. The change in hydrogen content occurs at discrete pressures

'Geophysical Laboratory, Carnegie Institution of Washington, Washington DC, USA, ${ }^{2}$ Consortium for Advanced Radiation Sources, University of Chicago, Chicago, Illinois, USA, ${ }^{3}$ HPCAT, Carnegie Institution of Washington, Advanced Photon Source, Argonne, Illinois, USA. *e-mail: zulu@gl.ciw.edu 


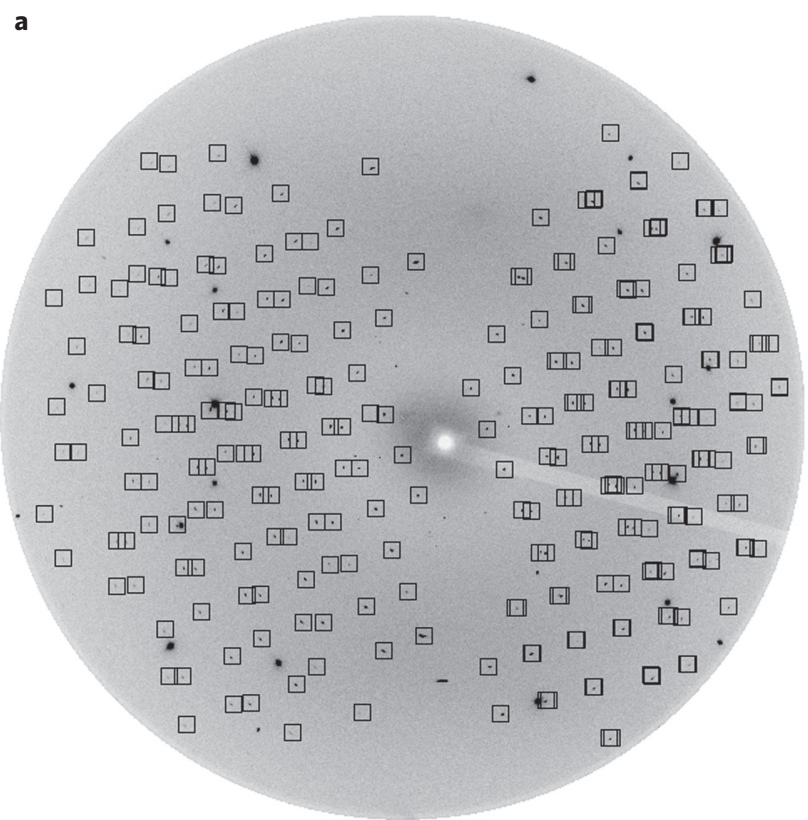

b

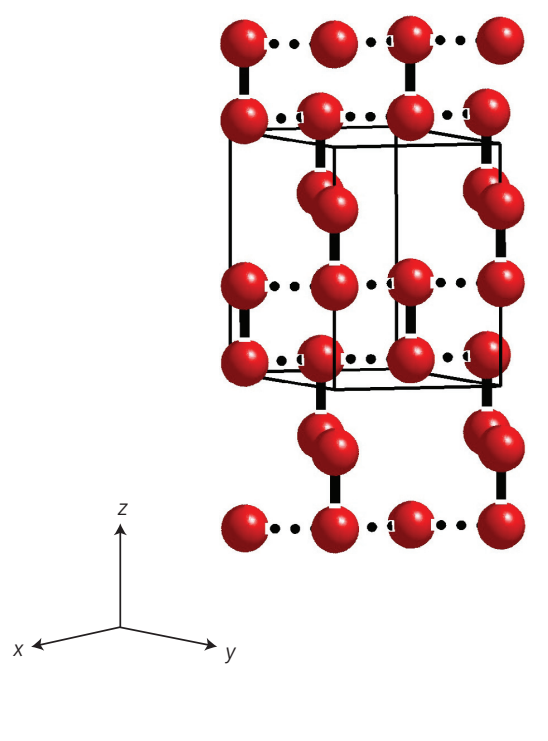

Figure 1 | X-ray single-crystal diffraction of $\mathrm{Xe}^{-\mathrm{H}_{2}}$ compound. a, Oscillation photograph of a single crystal of the Xe- $\mathrm{H}_{2}$ compound obtained at $4.9 \mathrm{GPa}$ (see Supplementary Information for details of indexing). b, Corresponding structure of the xenon sublattice deduced from these data.
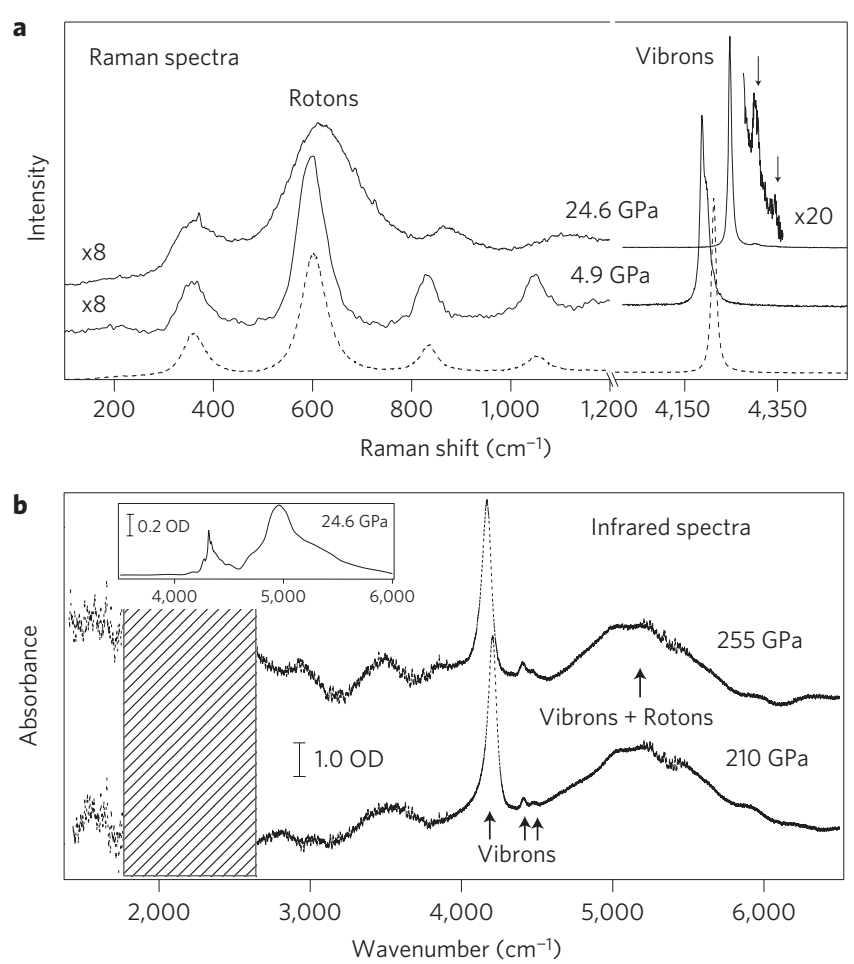

Figure 2 | Raman and infrared spectra of $\mathbf{X e}-\mathrm{H}_{2}$. a,b, Representative Raman spectra (a) and IR spectra (b) of the $\mathrm{Xe}-\mathrm{H}_{2}$ compound. The Raman spectrum of pure $\mathrm{H}_{2}$ at $4.9 \mathrm{GPa}$ is shown in a as a dotted curve. The vibron spectrum of the $\mathrm{Xe}-\mathrm{H}_{2}$ compound shows a multiplet structure that becomes evident at higher pressures, in contrast to the behaviour of pure $\mathrm{H}_{2}$. The lowpressure IR spectrum is shown in the inset of $\mathbf{b}$. The IR spectra at the highest pressure show no evidence of Drude absorption. The small increase at the longest wavelengths is indicative of diffraction effects due to decreasing sample size. Transmission at the longest wavelengths places an upper bound on the possible carrier density, with a corresponding upper bound for the plasma frequency of $0.2 \mathrm{eV}$ (ref. 29). The frequency range of the high twophonon absorption of the diamond is blocked.
(4.9 and $5.4 \mathrm{GPa}$ ), and there is no evidence for major changes in stoichiometry at higher pressures based on the continuity of the vibrational frequency shifts to $255 \mathrm{GPa}$ and the volume compression obtained from X-ray data to $50 \mathrm{GPa}$. The hydrogen content was bounded at these pressures based on the equations of state of the component elements Xe (ref. 16) and $\mathrm{H}_{2}$ (ref. 20). The space group $R 3$ constrains the number and placement of $\mathrm{H}_{2}$ molecules in the compound formed at $4.9 \mathrm{GPa}$. The observed unit cell volume of $801.4 \AA^{3}$ is $\sim 2 \%$ smaller than the sum $6 \mathrm{Xe}+42 \mathrm{H}_{2}$, suggesting a stoichiometry $\mathrm{Xe}\left(\mathrm{H}_{2}\right)_{7}$ for this phase. In comparison, the observed volume of $831.7 \AA^{3}$ at 7.1 GPa suggests a stoichiometry $\mathrm{Xe}\left(\mathrm{H}_{2}\right)_{8}$ for all pressures above $5.4 \mathrm{GPa}$. The systematic absences observed at $7.1 \mathrm{GPa}$ suggest that the space group changes from $R 3$ to $P 3$, with the accompanying change in hydrogen stoichiometry. Simulated annealing reverse Monte-Carlo calculations $s^{21,22}$ were performed with the diffraction intensity data used in the single-crystal refinement to further constrain the $\mathrm{H}_{2}$ positions. Xenon atoms were fixed at the positions (in $R 3$ ) obtained from the original refinement, and $\mathrm{H}_{2}$ locations were optimized from an initial random placement. Simulations with $42 \mathrm{H}_{2}$ molecules $\left(\mathrm{Xe}\left(\mathrm{H}_{2}\right)_{7}\right)$ yielded a structure that improved the refinement quality factor (in comparison to the model with only xenon atoms) $R_{1}$ by $0.5 \%$. The structure is shown in Fig. 3 . The assumption of $42 \mathrm{H}_{2}$ molecules in the unit cell agrees qualitatively with the highest peaks in the difference Fourier map obtained from a refinement that excluded hydrogen atoms.

The structural refinement provides direct information on the origin of the stability of this compound. Making use of the observed structure factors, we calculated the electron density maps. The single crystal at $4.9 \mathrm{GPa}$ was immersed in a hydrostatic liquid hydrogen medium, and the use of Boehler-Almax-type seats with an opening angle of $70^{\circ}$ allowed data to be collected over a large angular range. The resulting inherent high quality of the data and the span of the reciprocal space covered gave rise to electron density maps of very good quality. As described above, the xenon atoms are distributed as dimers rather than distinct atoms, with a uniform $\mathrm{Xe}-\mathrm{Xe}$ distance. Examination of the electron density maps shows no significant distortion of electron density between the xenon atoms at low pressures and therefore no interaction between them (Fig. 4a,b). However, a striking spread of electron 


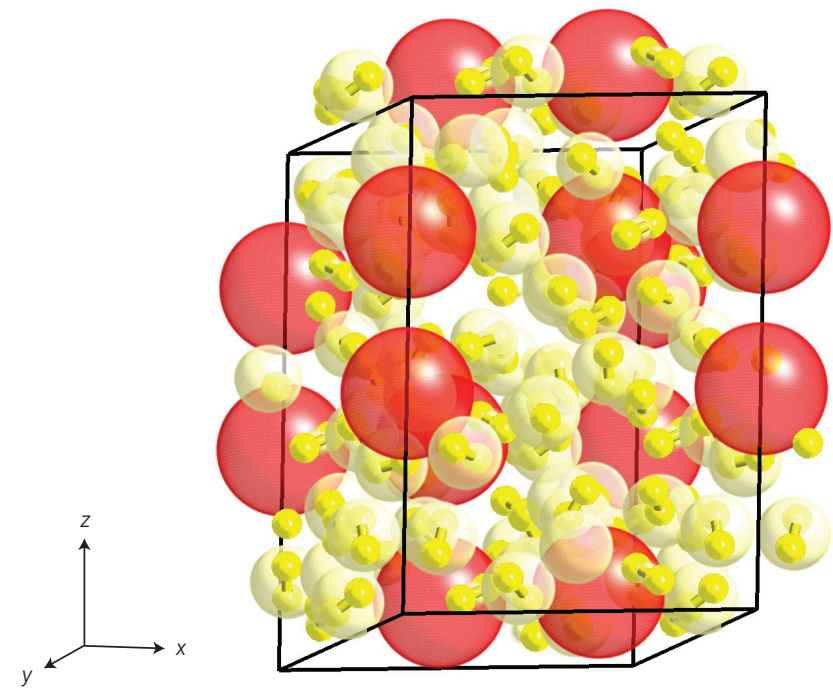

Figure 3 | Model structure of $\mathrm{Xe}\left(\mathrm{H}_{2}\right)_{7}$. The xenon atoms are surrounded by dumbbell-shaped, freely rotating hydrogen molecules represented by the spherical shells.

density into the interstitial space between the xenon atoms and from the $\mathrm{Xe}_{2}$ pairs towards the surrounding hydrogen molecules is observed at lower cutoff (Fig. 4c). We interpret these changes in electron density with pressure as arising from increasing chemical interaction between the xenon atoms in each pair as well as between $\mathrm{Xe}_{2}$ pairs and the surrounding array of hydrogen molecules. The spread of electron density from the xenon atoms (located on 3 a positions) to the surrounding hydrogen molecules serves to stabilize the $\mathrm{Xe}_{2}$ pairs by depopulating a fully filled $\sigma^{\star}$ molecular orbital of the $\mathrm{Xe}_{2}$ unit, creating a Xe-Xe bond. In xenonhalogen compounds, a gradual transition from van der Waals to covalent bonding between xenon atoms and the $\mathrm{X}_{2}$ halogen molecules is inferred and this depends on the extent of the overlap of the valence orbitals of Xe with the antibonding orbitals of the neighbouring molecule ${ }^{23}$. In a similar way, a gradual transition from a neutral $\mathrm{Xe}_{2}$ to an ionic $\mathrm{Xe}_{2}^{+}$seem to take place with pressure in the $\mathrm{Xe}-\mathrm{H}_{2}$ system $^{24}$. In other systems, this change in ionicity is initiated by either chemical means ${ }^{25}$ or xenon partial pressure ${ }^{26}$. Our data indicate that the $\mathrm{Xe}-\mathrm{Xe}$ bond length in the dimers attains the value observed in $\mathrm{Xe}_{2}^{+}$at $\sim 50 \mathrm{GPa}$. The formation of ionic xenon dimers is expected to give rise to charge transfer to the high-lying $\sigma^{\star}$ antibonding states of the $\mathrm{H}_{2}$ molecules, and results in additional weakening of the $\mathrm{H}-\mathrm{H}$ bond. Because the bond weakening (inferred from the observed decrease in vibrational frequencies) is similar to that found in pure $\mathrm{H}_{2}$ (ref. 18), the charge must be localized elsewhere (see Supplementary Information). This conclusion is consistent with the unusual spread in electron density distribution deduced from the refinement of X-ray data at lower pressures (Fig. 4c).

The lowest $\mathrm{H}_{2}$ vibron frequency decreases and shows a monotonic decrease with increasing pressure above $60 \mathrm{GPa}$. The decreasing vibron frequencies indicate a weakening of the covalent
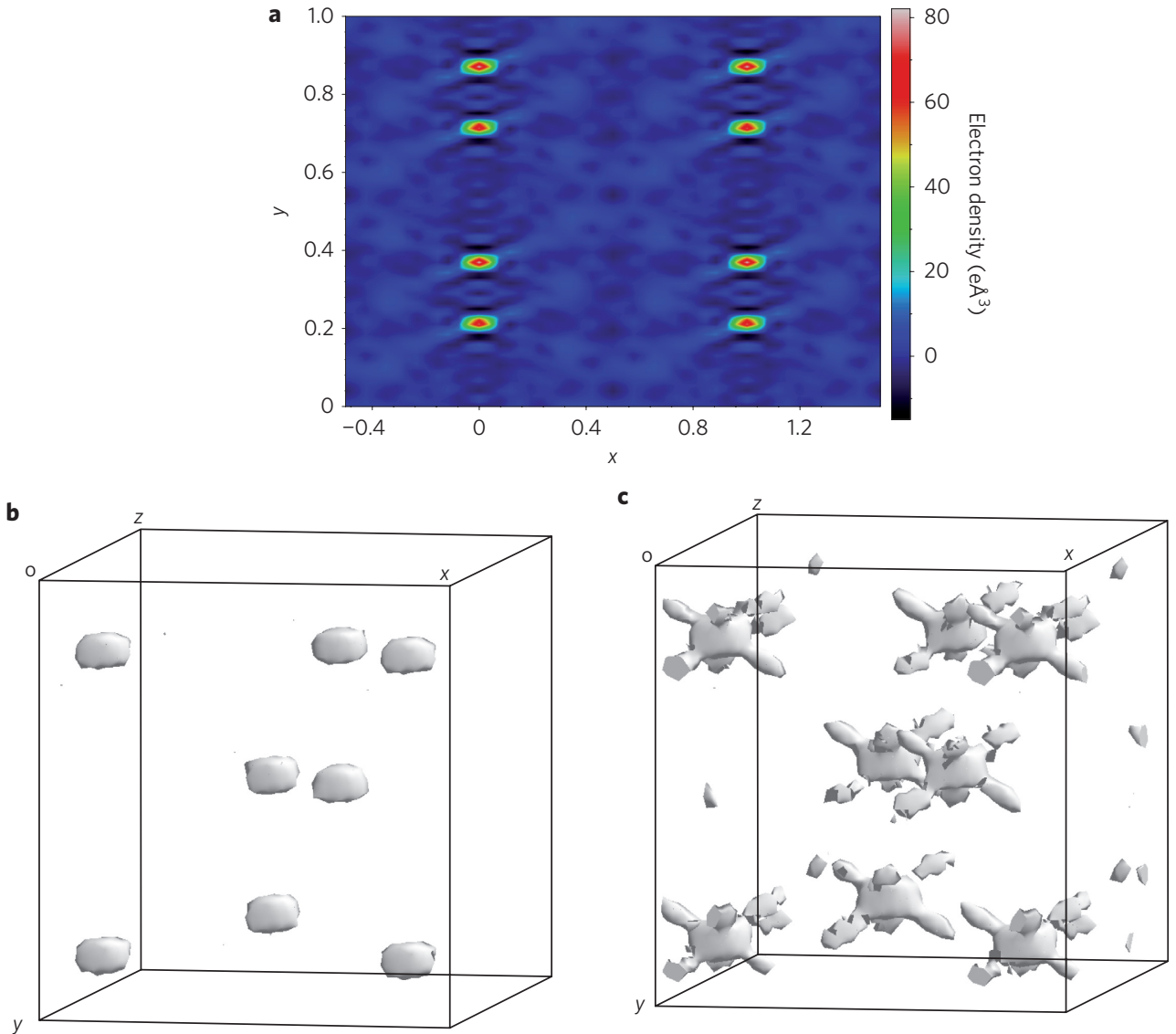

Figure 4 | Changes in the electron density of xenon. a, Electron density calculated from the observed structure factors (Beever-Lipson maps) ${ }^{30}$. The projection along $y$ obtained at the first level (a) shows the xenon pairs. b,c, Three-dimensional equal-energy contours obtained at different cutoff levels, $15 \mathrm{e}(\mathbf{b})$ and $5 \mathrm{e}(\mathbf{c})$, show the spread of electron density between the $\mathrm{Xe}$ atoms and in the direction of coordinated $\mathrm{H}_{2}$ molecules lying within the first coordination sphere of the $\mathrm{Xe}$ atoms. 
intramolecular bond at the highest pressures (that is, above $120 \mathrm{GPa}$ ), as observed in pure, solid hydrogen ${ }^{18,27}$. The observed vibron shift under pressure is similar to that found for $\mathrm{H}_{2}$ molecules dispersed in rare gas matrices under pressure ${ }^{28}$. The high-pressure compound formation reported here contrasts with the photochemically induced $\mathrm{Xe}-\mathrm{H}$ bond formation documented as impurities in cold rare gas matrices near ambient pressure ${ }^{9,10}$, because no spectroscopic evidence for the formation of $\mathrm{Xe}-\mathrm{H}$ bonds has been observed at any of the pressures studied. The IR measurements at the highest pressure show no evidence of Drude absorption $\left(\omega_{\mathrm{p}}<0.2 \mathrm{eV}\right)$ and therefore provide no indication of metallization (volume compression $V / V_{0} \approx 0.2$ ). Nevertheless, the measured variation of the structure on compression suggests the existence of xenon dimers forming a one-dimensional metal at still higher pressures. In addition to the fundamental interest in forming novel, hydrogenrich compounds as simple molecular quantum systems, the search for such new materials is important technologically (for example, in hydrogen storage); the unexpected stability of the compound described in the present study points to a new family of materials in hitherto unexplored regions of the temperature-pressurecomposition space.

Received 29 May 2009; accepted 15 October 2009; published online 22 November 2009

\section{References}

1. Crabtree, G. W., Dresselhaus, M. S. \& Buchanan, M. V. The hydrogen economy. Phys. Today 57, 39-44 (2004).

2. Hemley, R. J. Effects of high pressures on molecules. Ann. Rev. Phys. Chem. 51, 763-800 (2000).

3. Ashcroft, N. W. Metallic hydrogen: a high-temperature superconductor? Phys. Rev. Lett. 21, 1748-1749 (1968).

4. Ashcroft, N. W. Hydrogen dominant metallic alloys: high temperature superconductors? Phys. Rev. Lett. 92, 187002 (2004).

5. Carlsson, A. E. \& Ashcroft, N. W. Approaches for reducing the insulator-metal transition pressure in hydrogen. Phys. Rev. Lett. 50, 1305-1308 (1983).

6. Goettel, K. A., Eggert, J. H. \& Silvera, I. F. Optical evidence for the metallization of xenon at 132(5) GPa. Phys. Rev. Lett. 62, 665-668 (1989).

7. Reichlin, R. et al. Evidence for the insulator-metal transition in xenon from optical, X-ray and band-structure studies to $170 \mathrm{GPa}$. Phys. Rev. Lett. 62, 669-672 (1989).

8. Eremets, M. I., Gregoryanz, E. A., Struzhkin, V. V., Mao, H. K. \& Hemley, R. J. Electrical conductivity of xenon at megabar pressures. Phys. Rev. Lett. 85, 2797-2800 (2000).

9. Khriachtchev, L., Lignell, A., Juselius, J., Rasanen, M. \& Savchenko, E. Infrared absorption spectrum of matrix-isolated noble-gas hydride molecules: fingerprints of specific interactions and hindered rotation. J. Chem. Phys. 122, 14510-14517 (2005).

10. Khriachtchev, L., Pettersson, M., Runeberg, N., Lundell, J. \& Rasanen, M. A stable argon compound. Nature 406, 874-876 (2000).

11. Grochala, W. Atypical compounds of gases, which have been called 'noble'. Chem. Soc. Rev. 36, 1632-1655 (2007).

12. Wlodarczyk, A., McMillan, P. F. \& Greenfield, S. A. High pressure effects in anaesthesia and narcosis. Chem. Soc. Rev. 35, 890-898 (2006).

13. Vos, W. L. et al. A high-pressure van der Waals compound in solid nitrogenhelium mixtures. Nature 358, 46-48 (1992).
14. Loubeyre, P., Jean-Louis, M., LeToullec, R. \& Charon-Gérard, L. High pressure measurements of the He-Ne binary phase diagram at $296 \mathrm{~K}$ : evidence for the stability of a stoichiometric $\mathrm{Ne}(\mathrm{He})_{2}$ solid. Phys. Rev. Lett. 70, 178-181 (1993).

15. Hanni, H., Lantto, P., Runeberg, N., Jokisaari, J. \& Vaara, J. Calculation of binary magnetic properties and potential energy curve in xenon dimer: second virial coefficient of ${ }^{129} \mathrm{Xe}$ nuclear shielding. J. Chem. Phys. 121, 5908-5919 (2004).

16. Asaumi, K. High-pressure X-ray diffraction study of solid xenon and its equation of state in relation to metallization transition. Phys. Rev. B 29, 7026-7029 (1984).

17. Sears, D. R. \& Harold, P. K. Density and expansivity of solid xenon. J. Chem. Phys. 37, 3002-3006 (1962).

18. Mao, H. K. \& Hemley, R. J. Ultrahigh-pressure transitions in solid hydrogen. Rev. Mod. Phys. 66, 671-692 (1994).

19. Goncharov, A. F., Eggert, J. H., Mazin, I. I., Hemley, R. J. \& Mao, H. K. Raman excitations and orientational ordering in deuterium at high pressure. Phys. Rev. $B$ 54, R15590-R15593 (1996).

20. Loubeyre, P. et al. X-ray diffraction and equation of state of hydrogen at megabar pressures. Nature 383, 702-704 (1996).

21. LeBail, A. ESPOIR: a program for solving structures by Monte Carlo analysis of powder data. Mater. Sci. Forum 378-381, 65-70 (2001).

22. Brandenburg, K. \& Putz, H. Crystal Impact GbR, ENDEAVOR $1.6<$ http://www. crystalimpact.com/endeavor $>$ (2008)

23. Proserpio, D. M., Hoffman, R. \& Janda, K. C. The xenon-chlorine conundrum: van der Waals complex or linear molecule. J. Am. Chem. Soc. 113, 7184-7189 (1991)

24. Amarouche, M., Durand, G. \& Malrieu, J. P. Structure and stability of $\mathrm{Xe}_{\mathrm{n}}^{+}$clusters. J. Chem. Phys. 88, 1010-1018 (1988).

25. Drews, T. \& Seppelt, K. The $\mathrm{Xe}^{2+}$ ion-preparation and structure. Angew. Chem. Int. Ed. 36, 273-274 (1997).

26. Berry-Pusey, B. N., Anger, B. C., Laicher, G. \& Saam, B. Nuclear spin relaxation of ${ }^{129} \mathrm{Xe}$ due to persistent xenon dimers. Phys. Rev. A 74, 63408-63417 (2006).

27. Hanfland, M., Hemley, R. J., Mao, H. K. \& Williams, G. P. Synchrotron infrared spectroscopy at megabar pressures: vibrational dynamics of hydrogen to 180 GPa. Phys. Rev. Lett. 69, 1129-1132 (1992).

28. Loubeyre, P., LeToullec, R. \& Pinceaux, J. P. Raman measurements of the vibrational properties of $\mathrm{H}_{2}$ as a guest molecule in dense helium, neon, argon, and deuterium systems up to 40 GPa. Phys. Rev. B 45, 12844-12853 (1992).

29. Hemley, R. J., Mao, H.-K., Goncharov, A. F., Hanfland, M. \& Struzhkin, V. Synchrotron infrared spectroscopy to $0.15 \mathrm{eV}$ of $\mathrm{H}_{2}$ and $\mathrm{D}_{2}$ at megabar pressures. Phys. Rev. Lett. 76, 1667-1670 (1996).

30. Sheldrick, G. A short history of SHELX. Acta Cryst. Sec. A 64, 112-122 (2008).

\section{Acknowledgements}

The authors thank V. V. Struzhkin, G. Shen, Y. Meng and S. Sinogeikin for assistance and discussions. This work was supported by DOE-BES (DE-FG02-06ER46280), DOE-NNSA (CDAC), NSF-DMR (DMR-0805056), NSF-EAR (COMPRES) and the Balzan Foundation. A.P.S. is supported by DOE-BES under contract DE-AC02-06CH11357 and N.S.L.S. is supported by DOE-BES under contract no. DE-AC02-98CH10886.

\section{Author contributions}

M.S. and R.J.H. designed the project. M.S., A.F.G. and S.A.G. conducted the sample loading, spectroscopic studies and analysis. M.S., P.D., P.L., W.Y. and H.K.M. conducted the synchrotron X-ray diffraction measurements and analysis. Z.L. performed the synchrotron IR measurements. M.S., P.D., R.J.H., A.F.G. and S.A.G. wrote the manuscript.

\section{Additional information}

The authors declare no competing financial interests. Supplementary information accompanies this paper at www.nature.com/naturechemistry. Reprints and permission information is available online at http://npg.nature.com/reprintsandpermissions/. Correspondence and requests for materials should be addressed to M.S. 